\title{
Head Geometry Effects on Pneumatic Three-Hole Pressure Probes for Wide Angular Range
}

\author{
K M Argüelles Díaz, J M Fernández Oro and E Blanco Marigorta
}

University of Oviedo, Fluid Mechanics Group, Campus de Viesques, 33271, Gijón (Asturias), Spain

Email: arguelleskatia@uniovi.es

\begin{abstract}
Recent investigations [1] have demonstrated that it is possible to increase the operative angular range of pneumatic three hole pressure probes (THP) using a two-zoned method in the data reduction procedure. In this study, the influence of the head geometry on the performance of this type of probes is addressed, especially in the extended regions of the angular range, previously unconsidered in the literature. Three different geometries have been built to carry out the analysis, corresponding to cylindrical, trapezoidal and cobra type probes, with a separation angle of $60^{\circ}$ between the holes. Additionally, cylindrical and cobra type probes have been manufactured with angular distances of $30^{\circ}$ and $45^{\circ}$ between the holes, in order to advance the effect of the construction angle on the probes performance. The uncertainty transmitted from the measurements to the resultant flow variables has been also addressed. Moreover, the sensitivity of the different head designs to variations in the Reynolds number or deviations of the flow with respect to the measurement plane of the probe (pitch misalignments) have been concerned.

Major conclusions point out that cobra type probes provide the largest operative angular range, while cylindrical designs minimize the errors in the determination of both pressure and velocity flow variables, when the Reynolds number or the pitch angle differ from the baseline calibration.
\end{abstract}

Keywords: three-hole pressure probe, flow measurement, calibration, head geometry, pneumatic probe

\section{Introduction}

This paper is focused on the influence of the head geometry on the operative angular range of pneumatic THP probes. Also, the effect of these geometric differences on the errors introduced when retrieving the flow variables is of interest. In the literature, there are several references regarding the performance of a wide number of designs employed to build THP probes ([2]-[5]). All of them are limited to a reduced angular range $\left( \pm 30^{\circ}\right)$ that is obtained with the traditional calibration ([4], [5]). However, an extended angular range can be obtained if an alternative data reduction procedure is applied. Hence, the analysis for the extended angular range (from $\pm 30^{\circ}$ on) is the one presented here.

Pressure probes are instruments to measure the local velocity and the pressure value in a flow. Using three-hole devices, two-dimensional flows can be completely determined, while four (at least) or more holes are necessary to characterize three-dimensional velocity fields ([6]-[9]). They can be operated in nulling ([10], [11]) or non-nulling ([12], [13]) mode. Both methods require the pressure to be measured in the three holes of the probe simultaneously. The main difference is that the non-nulling mode requires a calibration method and a data reduction procedure, though the operating time is significantly reduced. Therefore, it is feasible to characterize unsteady flows ([14], [15]) or even turbulence ([16], [17]) if miniature pressure sensors with high frequency response are incorporated to the probe design. Then, pressure 
probes can be fast-response (dynamic) ([18]-[20]) or slow-response (pneumatic) type. Additionally, the head design can be very diverse: spherical, cylindrical, conical, cobra type, trapezoidal, wedge-shaped, etc. Depending on its particular geometry, probes will provide different operative performances. In this paper, pneumatic THP probes with typical head geometries (cylindrical, trapezoidal and cobra type) operated in the non-nulling mode are considered for the study. Figure 1 shows the maximum angular range that can be obtained for THP probes as a function of its head geometry and the data reduction procedure employed.

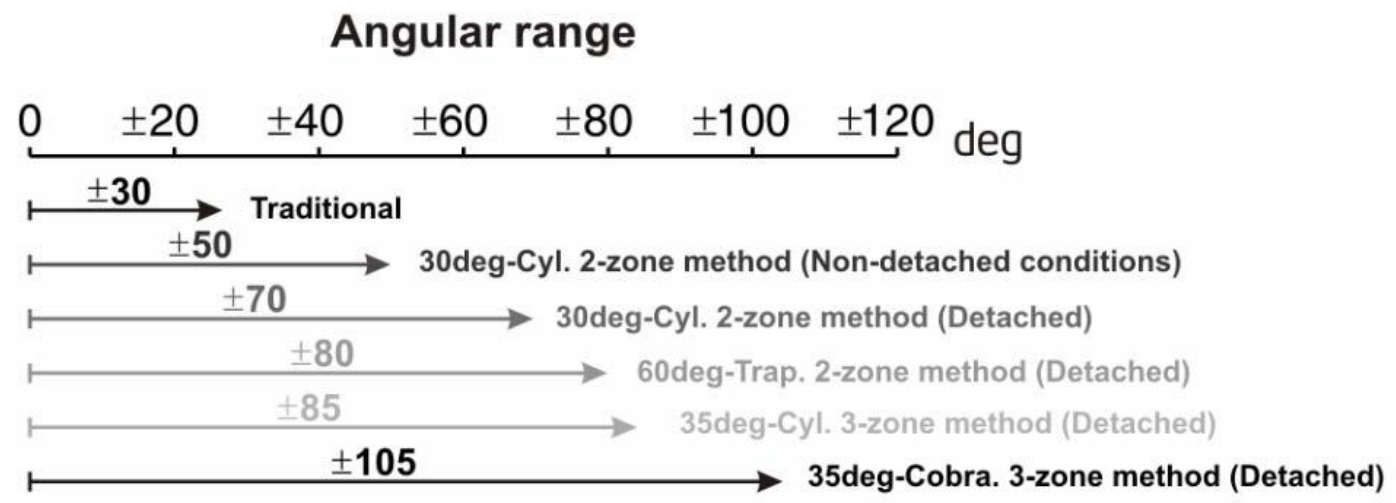

Figure 1. Angular ranges of THP probes as a function of the head geometry and the data reduction method employed.

Most references in the literature about THP probes report that the typical angular range is about $\pm 30^{\circ}$. However, this limit is not a consequence of a physical restriction of the probe operational principle, but fixed by the mathematical procedure employed to reduce the data. Using such procedure, usually known as "traditional calibration method", the calibration coefficients are undefined around $+37^{\circ}$ and $-37^{\circ}$. These two singular points lead to a practical reduction of the operative angular range into a $\pm 30^{\circ}$ interval. The calibration coefficients of the traditional method are:

$$
C_{\alpha}(\alpha)=\frac{P_{2}-P_{3}}{P_{1}-0.5\left(P_{2}+P_{3}\right)} ; \quad C_{P_{0}}(\alpha)=\frac{P_{0}-P_{1}}{P_{1}-0.5\left(P_{2}+P_{3}\right)} ; \quad C_{P_{S}}(\alpha)=\frac{P_{0}-P_{S}}{P_{1}-0.5\left(P_{2}+P_{3}\right)}
$$

where $P_{1}, P_{2}, P_{3}$ correspond to the pressures measured in every hole, $P_{0}$ and $P_{S}$ are the total and static pressures, $\alpha$ is the yaw flow angle and $C_{\alpha}, C_{P_{0}}$ and $C_{P_{S}}$ represent, respectively, the so-called angular, total pressure and static pressure coefficients.

Recent investigations by the authors [1] have revealed that singular points are not a real limitation of the angular range. It could be avoided discriminating different zones over the whole angular range of the flow, and defining for each zone calibration coefficients without singular points within it. This is the so-called "zone-based method". As a consequence, the angular range of THP probes can be extended until a real physical limitation arises: either double points in the calibration coefficients or repeated discrimination zones. In the first case, the angular coefficient is no more a monotonous function of the flow angle, so such angle cannot be univocally determined. In the second case, we can no more discriminate the particular angular interval requiring a specific calibration coefficient. It will be shown that, depending on the head geometry and the construction angle, sometimes the operative angular range is limited by double points, while others is restricted by the appearance of repeated zones.

Figure 2 shows the pressure coefficients $f_{i}(\alpha)(i=1,2,3)$ for a cylindrical probe with a $30^{\circ}$ construction angle, and the consequent angular calibration coefficient obtained with the traditional method. The subscript 1 refers to the central hole, while subscripts 2 and 3 
correspond to the left and right holes respectively. The coefficient $f_{1}(\alpha)$ has been taken from the experimental data provided by Weidman [21] for a Reynolds number of $2.3 \times 10^{4}$. The distributions shown in figure 2 can be considered as pseudo-theoretical, because the pressure coefficients in the lateral holes have been obtained shifting $\pm 30^{\circ}$ the central distribution. The limits of the angular range are included in the figure, both mathematical (singular points) and physical (repeated zones and double points). It is clearly observed that the angular coefficient of the traditional calibration is restricted by singularities. However, for this particular probe the maximum angular range is limited by the existence of repeated zones, reaching up to $\pm 85^{\circ}$ approximately. More details can be found in [22].

Figure 2. Mathematical and physical limitations of the angular range of a cylindrical probe with a $30^{\circ}$ construction angle.

Though the general rule is that higher the number of zones considered (up to six are possible using a sorting criterion for the measured pressures in the three holes) higher the attainable angular range, we have considered only a two-zone method to analyze the effect of the head geometry on the probe performance. Typically, a two-zone method is sufficient to provide a significant enlargement of the angular range, far beyond the maximum angular variations of any academic flow of research interest. In particular, we have employed the following two-zone coefficients:

$$
\begin{aligned}
& C_{\alpha}(\alpha)=\frac{P_{2}-P_{3}}{P_{1}+P_{2}-2 P_{3}} ; \quad C_{P_{0}}(\alpha)=\frac{P_{0}-P_{1}}{P_{1}+P_{2}-2 P_{3}} ; \quad C_{P_{S}}(\alpha)=\frac{P_{0}-P_{S}}{P_{1}+P_{2}-2 P_{3}} \text { when } P_{2}>P_{3} \\
& C_{\alpha}(\alpha)=\frac{P_{2}-P_{3}}{P_{1}+P_{3}-2 P_{2}} ; \quad C_{P_{0}}(\alpha)=\frac{P_{0}-P_{1}}{P_{1}+P_{3}-2 P_{2}} ; \quad C_{P_{S}}(\alpha)=\frac{P_{0}-P_{S}}{P_{1}+P_{3}-2 P_{2}} \text { when } P_{3}>P_{2}
\end{aligned}
$$


Notice that the pressure values measured in the holes are used to discriminate the different zones. This is the only direct criterion that can be employed, because the flow angle $\alpha$ is unknown a priori. Figure 3 compares the calibration coefficients defined with the traditional and the two-zone method for this particular cylindrical probe. The traditional angular range comprises $\pm 30^{\circ}$, while the extended angular range is about $\pm 70^{\circ}$.
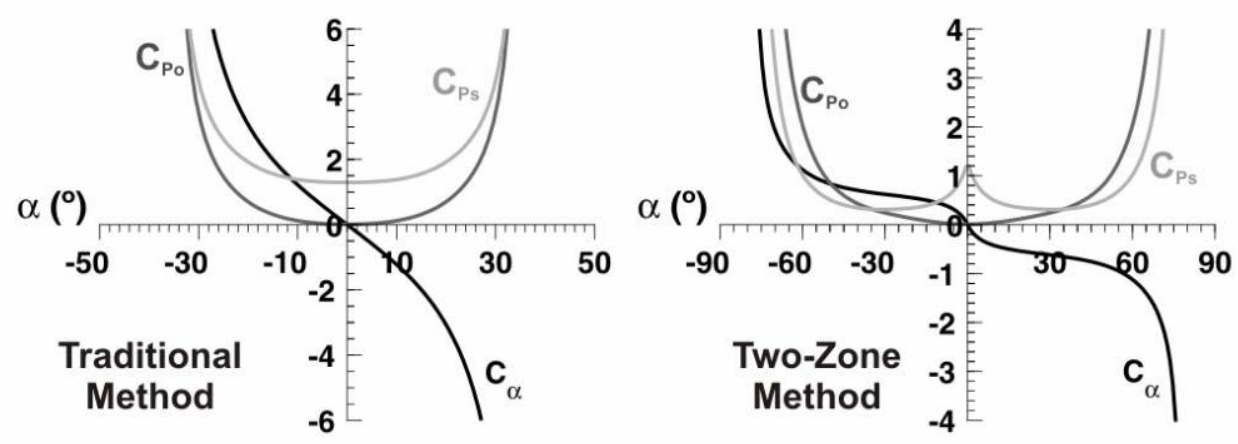

Figure 3. Comparison of the traditional and two-zone calibration coefficients for a cylindrical probe with a construction angle of $30^{\circ}$.

Following, the THP probes considered in this paper, as well as the calibration setup used for the measurements are presented. An investigation of the head geometry effects over the extended flow angle range is carried out. For that purpose, a detailed analysis of the pressure coefficients distributions in the probe holes is developed. Also, the influence of the angular distance between the holes (construction angle $\delta$ ) over the measurement range is studied. Next, the uncertainty transmitted to the flow variables as a function of both head geometry and construction angle is analyzed. Finally, the probes sensitivity to variations of the Reynolds number and misalignments in the pitch angle $\beta$ is also considered. To that end, several sets of measurements have been acquired in the calibration facility modifying these parameters. A detailed examination of the pressure coefficients in the holes is then conducted, obtaining the mean absolute value of the error introduced in the determination of the yaw angle.

\section{Probe Geometries and Experimental Setup}

To complete this investigation, three pneumatic probes with different head geometries have been built: cylindrical, trapezoidal and cobra type. All of them have been designed for a separation angle of $60^{\circ}$ between the holes (construction angle $\delta$ ). In the case of the cylindrical probe the construction angle is the angle defined between the central and the lateral holes, while for the trapezoidal probe is the angle formed between its frontal faces and for the cobra type is the skew angle of the lateral holes. Afterwards, cylindrical and cobra type probes have also been mechanized with angular distances of $30^{\circ}$ and $45^{\circ}$ between the holes, in order to study the influence of the construction angle in the measurement range. Figure 4 shows a sketch with the main geometrical parameters of the probes, as well as the flow angle $\alpha$. In the case of cylindrical probes the pressure variations in the holes with the flow angle are smooth, while both trapezoidal and cobra type geometries present sharp edges where the flow can be easily detached ([23], [24]).

The cylindrical probe has a $24 \mathrm{~mm}$ diameter head, with the three holes $(0.8 \mathrm{~mm}$ diameter $)$ placed at $16 \mathrm{~mm}$ from the semi-spherical tip. The diameter of the internal tubing of the probe is $1 \mathrm{~mm}$, for a total length of $0.5 \mathrm{~m}$, and the diameter of the support is $8 \mathrm{~mm}$. The trapezoidal probe has been built mechanizing all the faces (frontal and lateral) from a raw, solid piece of aluminium. Its frontal section was fixed to $6 \times 4 \mathrm{~mm}^{2}$. Following, three small holes (compared to the total area of the faces) of $0.5 \mathrm{~mm}$ diameter have been drilled on the faces. Finally, the cobra type probe is composed of three welded tubes of aluminium, with external and internal diameters of 2 and $1.5 \mathrm{~mm}$, respectively. The holes are covering all the available measuring 
surface, so its frontal section is $6 \times 2 \mathrm{~mm}^{2}$. The support, perpendicular to the measuring plane, has a $6 \mathrm{~mm}$ diameter, assembled $40 \mathrm{~mm}$ away from the probe frontal section.

In all the cases, the probes are connected to the pressure transducers through intermediate pneumatic tubings of $2 \mathrm{~m}$ length and $4 \mathrm{~mm}$ of internal diameter. The transducers employed in the calibration facility are Validyne DP15, with a $350 \mathrm{~mm} \mathrm{H}_{2} \mathrm{O}$ measurement range and an overall precision of $\pm 0.25 \%$. Output data from these transducers, after amplification, are acquired with a PCI 12 bits A/D card.
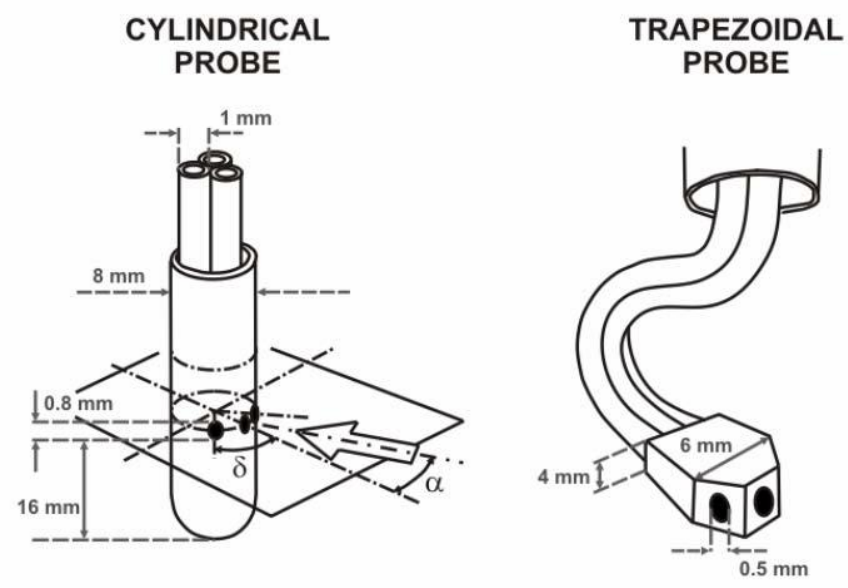

COBRA PROBE

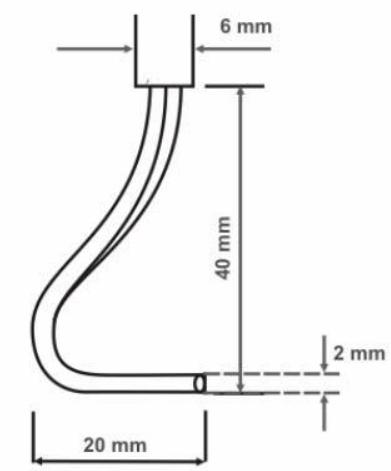

Figure 4. Probe geometries: cylindrical, trapezoidal and cobra type. Construction angle $\delta=60^{\circ}$.

The calibration facility is a small wind tunnel, operated with a centrifugal fan installed at the inlet. A settling chamber and a 4:1 contraction nozzle are placed upstream of the test section to ensure a uniform incident flow over the probes. The test section is an opened working area of $0.15 \times 0.30 \mathrm{~m}^{2}$, where the static pressure is thus atmospheric. Maximum velocity magnitude in the facility is $65 \mathrm{~m} / \mathrm{s}$, with a turbulence level around $0.5 \%$. The velocity magnitude is determined with a Pitot-static probe measuring in the test section, and also referred to the pressure established in the settling chamber. Both measurements are monitored with pressure transducers and checked on-line with U-manometers. The uncertainty for the mean velocity is estimated to be lower than $0.2 \%$. The probes are held in a rotating support and driven by two step motors, so they can be axially and radially rotated $360^{\circ}$ with a precision higher than $0.1^{\circ}$ in both directions. A sampling frequency of $1 \mathrm{kHz}$ per channel was employed for all the measurement sets. It is considered that this election is enough accurate for pneumatic probes with slow frequency response. Also, several filters are introduced, in both the electronic hardware and the digital processing of the signals, to avoid aliasing and filter out disturbance frequencies.

\section{Extended Angular Range}

This section analyzes the probes performance in the extended angular range. Such range is expected to be $\pm 80^{\circ}$ for the cobra type probe, $\pm 70^{\circ}$ for the trapezoidal geometry and $\pm 65^{\circ}$ for the cylindrical design. The three probes, presenting the same construction angle $\left(\delta=60^{\circ}\right)$, have been calibrated for the same Reynolds number $\left(1.8 \times 10^{4}\right)$ - referred to the local flow velocity and characteristic diameter of the probe head - and a zero-degree pitch angle. Besides, all the measurements taken with the probes are processed using the same data reduction procedure to retrieve the flow variables. This means that the differences observed in the angular range must be basically derived from the particular shape of the pressure coefficients distributions in the probes holes, that is, they will be a direct consequence of the differences between their head geometries. 
Figure 5 shows the distributions $f_{i}(\alpha)$ for the three holes of the probes. In the case of the cylindrical geometry, the pressure coefficients are practically the same for all the holes; the lateral distributions can be assumed to be approximately the central one shifted the construction angle (just manufacturing imperfections could lead to slight differences between them). For trapezoidal and cobra type probes the distributions are wider, with the lateral holes differing substantially from the central one. This implies that the limits due to double points (points P, Q and $\mathrm{R}$ in the figure) are more separated, so trapezoidal and cobra type geometries must cover a larger angular range than the cylindrical one. On the contrary, because $f_{i}$ distributions are wider their slopes are moderate, leading to higher uncertainties, as discussed later. Maximum pressure values in the lateral holes of the cylindrical probe appear at flow angles matching with the construction angle, i.e., reaching maxima for their corresponding zero-incidence angles $\left( \pm 60^{\circ}\right)$. Moreover, minimum values in the central hole are coincident with the lateral maxima. Conversely, trapezoidal and cobra type geometries present significant deviations of the maximum values, shifted towards lower angles (about $\pm 55^{\circ}$ ). In this case, maximum pressure values are no more coincident with the lateral maxima. Points marked in figure 5 as A and B indicate the limit due to duplicated zones in the operative angular range of the cylindrical probe. The other two geometries are not reaching such limit throughout the $\pm 120^{\circ}$ interval.

Figure 5. Distributions of the pressure coefficients in the three holes of cylindrical, trapezoidal and cobra type probes, $\delta=60^{\circ}$.

To highlight the existing differences between the three geometries, two graphics introducing the distributions of the pressure coefficients in the central and the left holes are reproduced in figure 6. For both holes, the minimum pressure values are always lower for the trapezoidal probe. This effect is a direct consequence of the losses associated to the flow separation developed in the wedge edges of the probe. Absolute values of the flow angle experiencing minimum pressure values are always lower for the cylindrical geometry. In the case of the central holes, minima of the pressure coefficient represent the double points limit; while in the lateral holes indicate that detached conditions of the flow are set off. Therefore, concerning both limitations for the angular range, the cobra type probe demonstrates better performance. For zero-incidence flow $\left(\alpha=0^{\circ}\right)$, the pressure coefficients in the lateral holes are negative for the cylindrical probe, but positive for the trapezoidal and cobra type designs. Both cobra type and trapezoidal probes present characteristic disturbances between $0^{\circ}$ and $30^{\circ}$ (the same behaviour is observed for the right hole between $0^{\circ}$ and $-30^{\circ}$ ) due to partial detached conditions of the flow, induced by the wedge edges of their head geometry. In addition, the region with total separation takes place from $30^{\circ}$ on $\left(-30^{\circ}\right.$ for the right hole $)$ - the flow is aligned with the lateral face in the trapezoidal and cobra type probes -. Beyond this transition zone, in the fully-detached region, the pressure coefficient of the trapezoidal probe is significantly lower than that of the other two geometries. It is observed that this total separation is smoother in the case of the cobra type probe. 
Moreover, the trapezoidal probe turns to be unstable when large flow angles are measured, and the cylindrical design is susceptible to Von Kárman vortex shedding. It is believed that the better performance of the cobra type probe for large flow angles is due to the relative size of the lateral holes; the pressure transducer is measuring a kind of averaged value of the detached conditions on the overall surface.

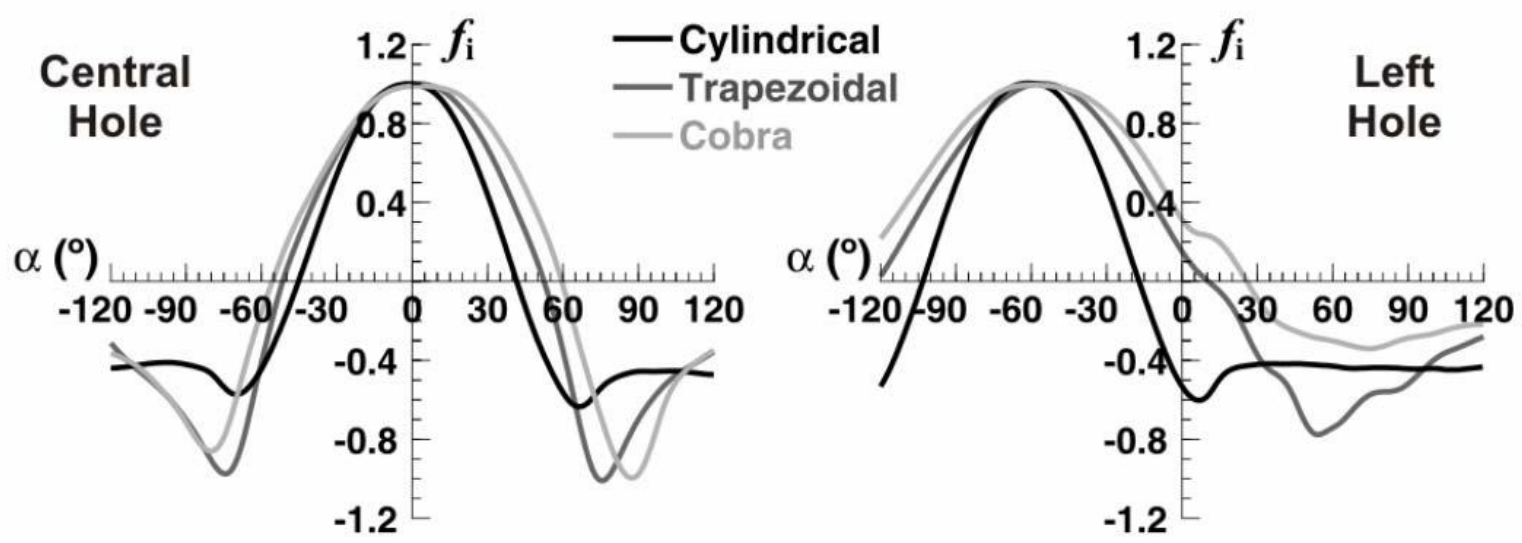

Figure 6. Distributions of the pressure coefficients in the central and left holes of cylindrical, trapezoidal and cobra type probes, $\delta=60^{\circ}$.

Figure 7 compares the angular coefficient $C_{\alpha}$ that is obtained after applying the two-zone method to reduce the data measured with the three considered probes. $C_{\alpha}$ values range between \pm 1.1 for the cylindrical probe and between \pm 1.3 and \pm 2.4 for the trapezoidal and cobra type geometries, respectively. The three coefficients are zero-valued when $\alpha=0^{\circ}$, presenting antisymmetric features with respect such angle. Their slopes are relatively smooth for the whole angular range. This is because the limit of the two-zone calibration method is established by double points, instead of the singular points arising in the traditional method. The coefficient of the cylindrical probe presents no oscillations, while the other two geometries exhibit several local inflexion points around $\pm 30^{\circ}$ and $\pm 55^{\circ}$. As can be seen in figure 7, the largest angular range corresponds to the cobra type probe $\left( \pm 80^{\circ}\right)$, followed by the trapezoidal $\left( \pm 70^{\circ}\right)$ and the cylindrical one $\left( \pm 65^{\circ}\right)$, matching with the location of the double points marked in figure 5 .

Figure 7. Angular coefficients obtained with the two-zone method for cylindrical, trapezoidal and cobra type probes, $\delta=60^{\circ}$. 


\section{Construction Angle Effects}

Not only is the angular range of the probes affected by the head geometry, but also by the angular distance between the holes (construction angle $\delta$ ). This section addresses the influence of this parameter over the cylindrical and cobra type geometries, when operated at $\operatorname{Re}=1.8 \times 10^{4}$ and $\beta=0^{\circ}$.

Figure 8 shows the distributions of the pressure coefficients in the left hole of a cylindrical probe built with construction angles of $30^{\circ}, 45^{\circ}$ and $60^{\circ}$. The three corresponding angular calibration coefficients obtained with the two-zone method are also included in the figure. It is clearly observed that the distributions of the pressure coefficients are identical for all the construction angles. The only difference is that they appear shifted, presenting its maximum value when the flow angle matches with the construction one. Therefore, it is expected that the restriction due to repeated zones in the angular range will be firstly reached with lower distance between the holes. On the other hand, significant differences are found in the distributions of the angular coefficients shown in the right plot of the figure. In the case of $\delta=30^{\circ}$ and $45^{\circ}$ the distributions are not as uniform as in the $60^{\circ}$ case, with local inflexion points around $\pm 5^{\circ}$ and $\pm 40^{\circ}$. As a consequence, the slope of the angular coefficients within $\pm 5^{\circ}$ is stepper for $30^{\circ}$ and $45^{\circ}$ cylindrical probes. This leads to a lower uncertainty in the flow angle for that interval (shown later). About the same angular range $\left( \pm 65^{\circ}\right)$ is reached for both $45^{\circ}$ and $60^{\circ}$ designs, while for the $30^{\circ}$ construction angle the angular range is larger $\left( \pm 70^{\circ}\right)$. For this case the limit is imposed due to duplicated zones, while the other ones are restricted by the emergence of double points.

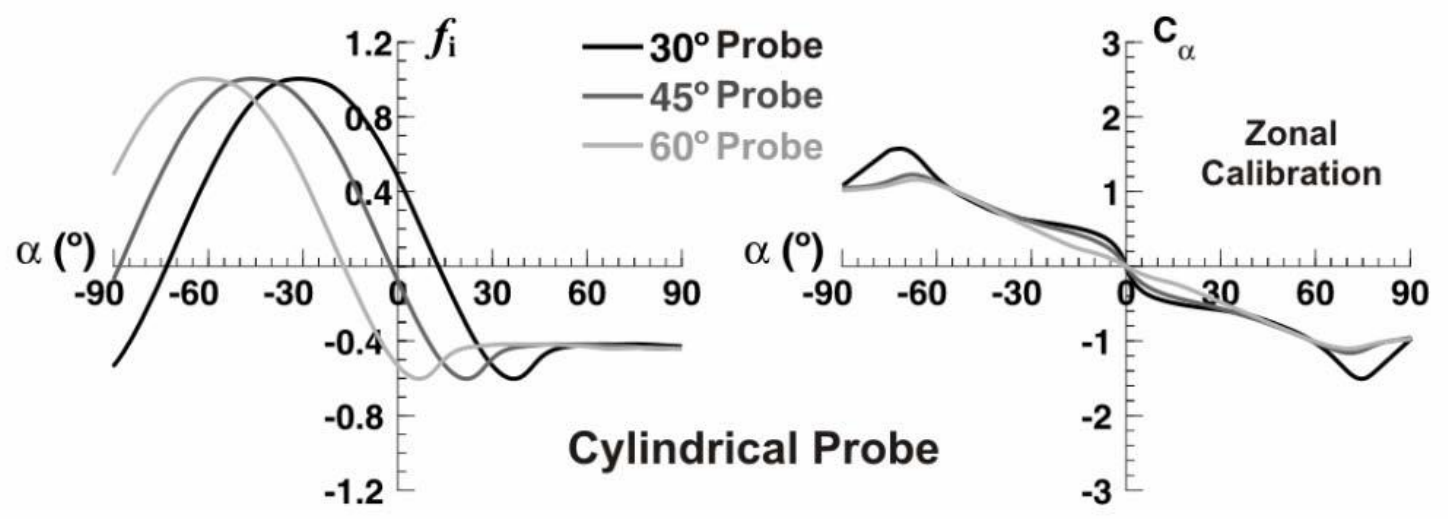

Figure 8. Pressure and angular coefficients for three different construction angles of a cylindrical probe, $\delta=30^{\circ}, 45^{\circ}$ and $60^{\circ}$.

Analogous to figure 8, the distributions of pressure coefficients for the left hole as well as the angular calibration coefficients obtained with the two-zone method are shown in figure 9 for the cobra type probe built with $30^{\circ}, 45^{\circ}$ and $60^{\circ}$ construction angles. Important differences are noticeable between the pressure coefficients. Maximum value is obtained for a flow angle matching with the construction angle only for $\delta=30^{\circ}$. For the remaining designs the maxima location are significantly deviated: about $\pm 35^{\circ}$ for $\delta=45^{\circ}$ and $\pm 55^{\circ}$ for $\delta=60^{\circ}$. Differences in the pressure coefficients are especially observed in the region where total flow separation is incipient $\left( \pm 30^{\circ}\right.$ for $\delta=60^{\circ}, \pm 45^{\circ}$ for $\delta=45^{\circ}$ and $\pm 60^{\circ}$ for $\delta=30^{\circ}$ ). This detachment seems to be less abrupt for the cobra type probe with $60^{\circ}$ construction angle. Therefore, major differences in the angular coefficients shown in figure 9 are developed from $\pm 45^{\circ}$ on, that is, fully within the extended angular range of cobra type probe. The measurement range is significantly modified when the angular separation of the holes changes: $\pm 80^{\circ}$ for $\delta=60^{\circ}, \pm 85^{\circ}$ for $\delta=30^{\circ}$ and even $\pm 95^{\circ}$ for $\delta=45^{\circ}$. For construction angles of $30^{\circ}$ and $45^{\circ}$, the limitation is due to the existence of singular points arising in the calibration coefficients of the two-zone method, while for $\delta=60^{\circ}$ the restriction derives from the appearance of double points. 


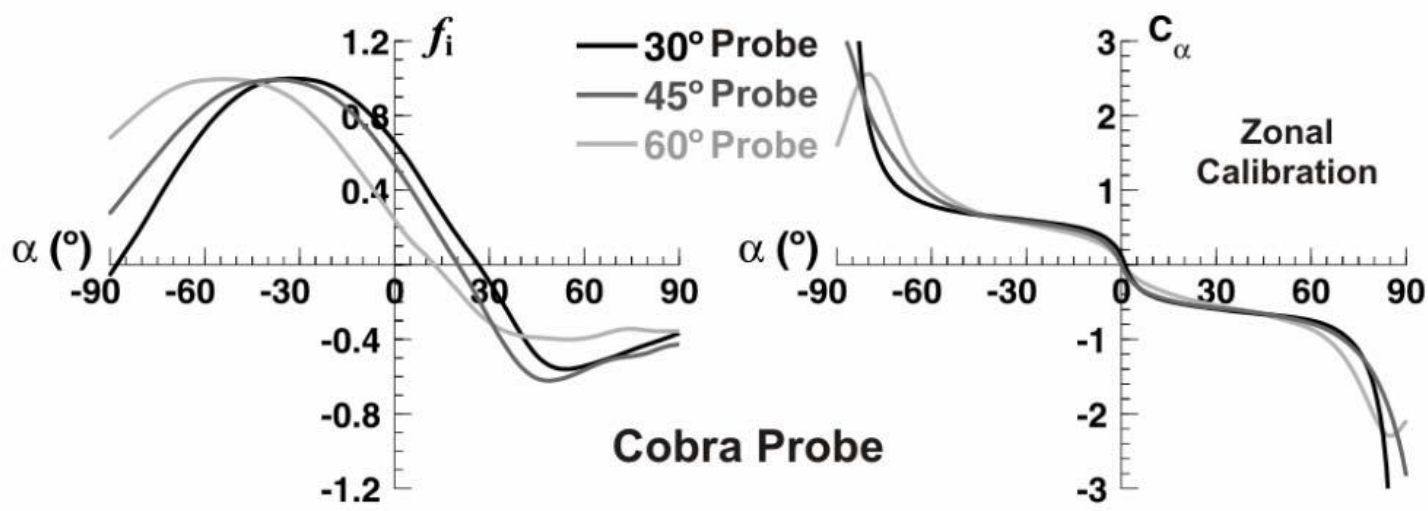

Figure 9. Pressure and angular coefficients for a cobra type probe with three different angular distances between the holes, $\delta=30^{\circ}, 45^{\circ}$ and $60^{\circ}$.

The maximum angular range for cylindrical and cobra type geometries has been plotted in figure 10 as a function of the angular distance between the holes. For completeness, also the one corresponding to the trapezoidal probe of $60^{\circ}$ construction angle has been added to the plot. It is possible to obtain the maximum angular range for any construction angle of a cylindrical probe, only displacing the pressure distributions of the lateral holes (ideally identical to the central one) according to the $\delta$ angle to be considered. Up to $\delta=35^{\circ}$ the limitation of the angular range of the cylindrical probe is due to the emergence of duplicated zones. If $\delta>35^{\circ}$ then the restriction is imposed by the arising of double points. For this particular geometry, the maximum measurement range is obtained for an angular distance between the holes of $35^{\circ}$. A detailed discussion of the influence of the construction angle in the attainable angular range of cylindrical probes can be found in [25].

For a cobra type probe, the maximum angular range is limited by the emergence of singular points in the calibration coefficients when $\delta=30^{\circ}$ and $45^{\circ}$. However, as previously discussed, this is not a real limitation, so an alternative definition of zones and calibration coefficients without singularities could provide an even larger angular range. Particularly, it has been demonstrated that a cobra type probe with a $35^{\circ}$ construction angle may provide a $\pm 105^{\circ}$ range when operated using a three-zone method [26]. This evidence has been also included in figure 10. On the contrary, when the angular distance between the holes is increased to $60^{\circ}$, the limitation for the angular range is now due to double points, so it is not possible to increase even more that range (independently of the data reduction method employed). The same is applicable for the $60^{\circ}$ trapezoidal probe.

Figure 10. Maximum angular range as a function of the probes construction angle. 


\section{Uncertainty Analysis}

In a pressure probe the transmission of uncertainty from the sensors to the flow variables is independent of the data reduction procedure employed [1]. It depends on the particular shape of the distributions of the pressure coefficients on the holes, which are especially sensitive to the head geometry and the construction angle. This section provides an analysis of the uncertainty transmission as a function of these two parameters. Once again, all the results correspond to $\operatorname{Re}=1.8 \times 10^{4}$ and $\beta=0^{\circ}$.

The uncertainty of the flow angle $I_{\alpha}$, of the dynamic pressure $I_{P_{d}}$, and of the static pressure $I_{P_{s}}$, are determined by the following expressions [1]:

$$
\begin{gathered}
I_{\alpha}=\frac{I_{p}}{P_{d}} \cdot \frac{\sqrt{\left(f_{3}-f_{2}\right)^{2}+\left(f_{1}-f_{3}\right)^{2}+\left(f_{2}-f_{1}\right)^{2}}}{f_{1}^{\prime}\left(f_{3}-f_{2}\right)+f_{2}{ }^{\prime}\left(f_{1}-f_{3}\right)+f_{3}{ }^{\prime}\left(f_{2}-f_{1}\right)} \\
I_{P_{d}}=I_{p} \cdot \frac{\sqrt{\left(f_{3}^{\prime}-f_{2}{ }^{\prime}\right)^{2}+\left(f_{1}^{\prime}-f_{3}{ }^{\prime}\right)^{2}+\left(f_{2}{ }^{\prime}-f_{1}{ }^{\prime}\right)^{2}}}{f_{1}^{\prime}\left(f_{3}-f_{2}\right)+f_{2}{ }^{\prime}\left(f_{1}-f_{3}\right)+f_{3}{ }^{\prime}\left(f_{2}-f_{1}\right)} \\
I_{P_{s}}=I_{p} \cdot \frac{\sqrt{\left(f_{2} f_{3}^{\prime}-f_{3} f_{2}{ }^{\prime}\right)^{2}+\left(f_{3} f_{1}^{\prime}-f_{1} f_{3}{ }^{\prime}\right)^{2}+\left(f_{1} f_{2}{ }^{\prime}-f_{2} f_{1}\right)^{2}}}{f_{1}{ }^{\prime}\left(f_{3}-f_{2}\right)+f_{2}{ }^{\prime}\left(f_{1}-f_{3}\right)+f_{3}{ }^{\prime}\left(f_{2}-f_{1}\right)}
\end{gathered}
$$

where the prime superscript stands for differentiation of the variable respect to the flow angle $\alpha$, $P_{d}$ represents the dynamic pressure and $I_{p}$ is the uncertainty in the pressure measurement, supposed identical for the three holes of a probe (same sensors in all the holes).

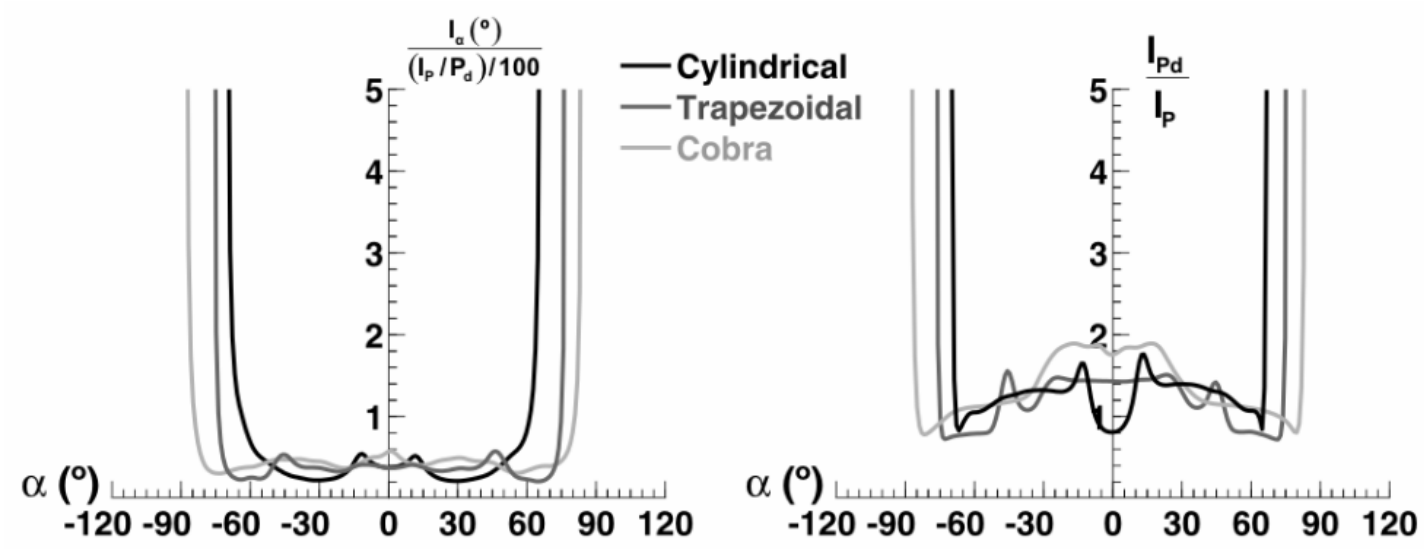

Figure 11. Uncertainty in the angle and dynamic pressure for cylindrical, trapezoidal and cobra type probes, $\delta=60^{\circ}$.

Figure 11 shows the uncertainty in the flow angle and the dynamic pressure (equations (4) and (5)) for the cylindrical, trapezoidal and cobra type probes with an angular distance of $60^{\circ}$ between the holes. The uncertainty in the flow angle (in degrees) is expressed as a percentage of the uncertainty of the pressure measurement $I_{p}$, relative to the dynamic pressure. The expression of the uncertainty in the dynamic pressure is related to $I_{p}$. For zero-incidence the uncertainty in the flow angle is the same for trapezoidal and cylindrical probes $\left(0.4^{\circ}\right)$, and a bit 
higher for the cobra type geometry $\left(0.6^{\circ}\right)$. The cylindrical geometry exhibits a significant increase around $\pm 10^{\circ}$, progressively mitigated until a $0.2^{\circ}$ value at $\pm 30^{\circ}$. In the case of the cobra type probe, the maximum value is obtained at zero incidence angle, with two local minima around $\pm 15^{\circ}$. In general, the trapezoidal probe shows a more uniform $I_{\alpha}$ distribution than the other two geometries in the range of the traditional calibration. In the extended angular range (from $\pm 30^{\circ}$ on), the cylindrical probe provides the lower uncertainty in the retrievement of the flow angle. Also, it can be said that, in general, the uncertainty is higher for the cobra type probe. Around $\pm 45^{\circ}$ the trapezoidal geometry reaches maximum values, losing the uniformity shown within the traditional measurement range.

At zero-incidence the lowest values of the uncertainty in the dynamic pressure correspond to the cylindrical probe, while the highest are encountered with the cobra type probe. This trend is maintained within the $\pm 15^{\circ}$ interval, where the cylindrical probe experiences a significant peak. In the extended angular range, it can be said that both cylindrical and cobra type probes present similar uncertainties. For the trapezoidal design an increase is observed around $\pm 45^{\circ}$. Afterwards, for this probe the uncertainty diminishes, reaching the lowest values for the rest of the angular interval. In any case, $I_{P_{d}}$ is never exceeding the value $2 I_{P}$ for all the flow angles, except in the boundaries of the angular range.

Results shown in figure 11 indicate that, in general, both trapezoidal and cobra type probes present a major transmission of uncertainty than the cylindrical geometry, as a direct consequence of the shape adopted by the distributions of their pressure coefficients, wider and with reduced slopes, as previously discussed in figure 5 .

Figure 12. Uncertainty in the angle corresponding to cylindrical and cobra type probes for different construction angles, $\delta=30^{\circ}, 45^{\circ}$ and $60^{\circ}$.

Figure 12 shows a combined representation of the uncertainty in the flow angle for a cylindrical and a cobra type probes with different construction angles $\left(30^{\circ}, 45^{\circ}\right.$ and $\left.60^{\circ}\right)$. In the left side of the figure, results corresponding to the cylindrical design have been represented and compared to those of the cobra geometry in the right side. Evidently, $I_{\alpha}$ distributions are symmetrical respect to $\alpha=0^{\circ}$. Within the extended angular range few differences are noticeable in the uncertainty of the flow angle when the construction angle of the cylindrical probe is varied. It is only of consideration the fact that between $-30^{\circ}$ and $-40^{\circ}$ the uncertainty for $\delta=30^{\circ}$ is higher. Maximum values of $I_{\alpha}$ are not exceeding $0.6^{\circ}$, except in the limits of the operative range. In the traditional angular range the lower uncertainty is obtained when the measurements are conducted with the $45^{\circ}$ cylindrical probe. For the cobra type probe larger differences are observed. In general, for the whole angular range, the uncertainty in the angle is increased when the construction angle is progressively reduced, exceeding $1^{\circ}$ when $\delta=30^{\circ}$. For a $60^{\circ}$ distance between the holes, $I_{\alpha}$ is lower with a much more uniform distribution. Maxima of uncertainty 
appear around $30^{\circ}$ and $45^{\circ}$ for construction angles of $45^{\circ}$ and $30^{\circ}$ respectively. Though not shown here, similar trends have been found for the uncertainty of the dynamic and static pressures. Summarizing, it can be said that the transmission of uncertainty is increased for both head geometries when the angular separation of the holes is reduced.

\section{Reynolds Number Effects}

It is well-known that the assumption of pressure coefficients $f_{i}$ depending exclusively on the flow angle and not on the velocity magnitude is only valid for a close range of Reynolds numbers. In the case of a cylindrical probe, this hypothesis is assumable for Reynolds numbers going from $1.0 \times 10^{4}$ to $1.0 \times 10^{5}$. Other types of probes present different valid intervals, depending on their particular geometries. A complete discussion of the Reynolds number effects on the calibration of five-hole probes is available in [27].

To establish the sensitivity of the pressure probes to changes in the flow velocity magnitude, a set of measurements has been acquired with the cylindrical, the trapezoidal and the cobra type geometries for eight Reynolds numbers ranging from $1.0 \times 10^{4}$ to $2.4 \times 10^{4}$. The three probes have a construction angle of $60^{\circ}$ and were operated for a zero-pitch angle.

\section{Trapezoidal Probe}

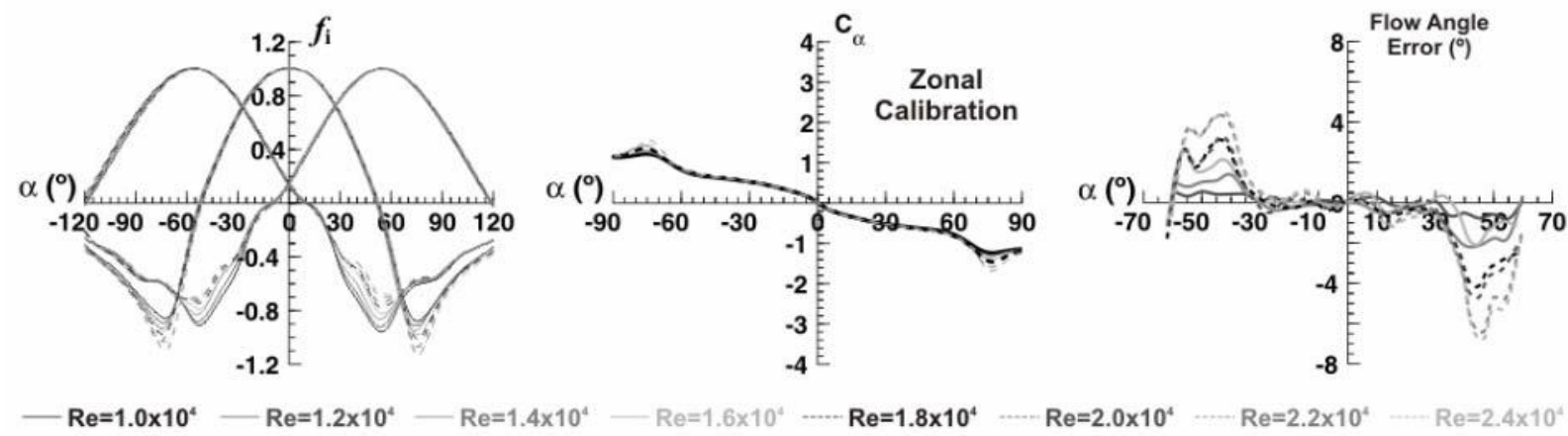

Figure 13. Pressure coefficients, angular coefficients and flow angle error as a function of the Reynolds number for a trapezoidal probe, $\delta=60^{\circ}$.

Figure 13 shows the results obtained with the trapezoidal geometry. The figure represents the distributions of the pressure coefficients in the three holes (left plot), the angular coefficients derived from the two-zone method (central plot) and the error introduced in the determination of the flow angle (right plot), for the eight Reynolds numbers. Within the traditional angular range the pressure coefficients are practically independent of the velocity magnitude. Its effect is much more evident in the extended angular range, especially in those regions where the flow is detached. An inverse trend is observed between central and lateral holes: in the central hole the minimum value of the pressure coefficient (around $\pm 75^{\circ}$ ) is reduced when the Reynolds number increases, whereas in the lateral ones (around $\pm 55^{\circ}$ ) increases. Consequently, for the angular coefficients (central plot) almost negligible differences are found in the traditional range. Only close to the practical limits of the extended range $\left( \pm 70^{\circ}\right)$ differences are more evident, with an increase of the $C_{\alpha}$ absolute value when the velocity magnitude increases.

Besides, it has been determined the error magnitude in the retrievement of the flow angle $\alpha$ when the Reynolds number is modified. To that end, the calibration corresponding to a $\mathrm{Re}=1.0 \times 10^{4}$ has been employed to reduce all data measured for the rest of the Reynolds numbers. The results are shown in the right plot of figure 13 for the trapezoidal design. Though the errors for the cylindrical and the cobra type probes are not explicitly analyzed here, the sensitivity to the Reynolds number is compared for the three geometries in forthcoming figure 15. Thus, the maximum angular interval considered to calculate the flow angle error corresponds to the lowest operative range of the three probes $\left( \pm 60^{\circ}\right.$, corresponding to the 
cylindrical geometry). The error in the flow angle determination is expressed as a deviation in degrees. In the range of the traditional calibration the errors are low, never exceeding $0.5^{\circ}$. In the extended intervals, it is clearly observed a progressive increase of the error as the difference between the real Reynolds number and the one of the baseline calibration enlarges. Important errors are noticeable, reaching up to $\pm 4.5^{\circ}$ when $\mathrm{Re}=2.4 \times 10^{4}$. These results suggest a marginal influence of the Reynolds number on the partial separation observed in the lateral holes between $0^{\circ}$ and $\pm 15^{\circ}$. On the contrary, fully-detached regions (from $\pm 30^{\circ}$ on) are notably influenced by variations in the Reynolds number, though they are not clearly reflected in the distributions of the angular coefficients (central plot). In terms of the Reynolds number influence, it is expected a better performance in the whole angular range for a trapezoidal probe with a construction angle of $30^{\circ}$ or $35^{\circ}$, due to the displacement of the fully-detached regions in the lateral holes towards the external intervals.

Figure 14. Pressure coefficients of the cylindrical and the cobra type probes as a function of the Reynolds number, $\delta=60^{\circ}$.

Figure 14 shows a combined representation of the pressure coefficients obtained with the cylindrical and cobra type probes when the Reynolds number is modified. The set of lines in the left side corresponds to the cylindrical probe, while the cobra results are plotted in the right side. Obviously, the coefficients are symmetric with respect the zero-incidence angle. As expected, the pressure distributions $f_{i}$ of the cylindrical geometry are unaffected by variations in the velocity magnitude. For the central hole of the cobra type probe major differences are observed from $\pm 80^{\circ}$ on: a reduction in the minimum value of $f_{1}$ overcomes when increasing the Reynolds number. On the contrary, for the lateral holes major differences are concentrated towards the central interval $\left( \pm 65^{\circ}\right)$. In this case, $f_{2,3}$ values are increased with higher velocity magnitudes.

Whatever, variations with the Reynolds number are less important than those of the trapezoidal design, especially in the region with large flow angles. Therefore, it is expected for the error in the determination of such angles to be lower for the cobra type probe than for the trapezoidal geometry.

To compare the sensibility of the three probes to variations in the Reynolds number, the mean absolute value of the flow angle error (in degrees) has been represented in figure 15. Obviously, the mean error corresponding to $\mathrm{Re}=1.0 \times 10^{4}$ is zero in all the cases. For the cylindrical probe the mean value is practically constant, not exceeding $0.5^{\circ}$ in all the regime set. For the cobra type probe the mean error is slightly higher than for the trapezoidal one until $\mathrm{Re}=1.6 \times 10^{4}$. For higher Reynolds numbers, this trend is inverted, due to the strong flow separations associated to the wedge edges of the trapezoidal geometry at higher velocities. In any case, the mean error is not exceeding $1.1^{\circ}$ for the cobra type probe, while in the case of the trapezoidal one it can be as 
high as $1.8^{\circ}$. Finally, it must be remembered that the results shown in figure 15 have been calculated for the operative angular range of the cylindrical design $\left( \pm 60^{\circ}\right)$. If a higher angular range would have been considered, an increment of the mean error would have been observed for trapezoidal and cobra type probes, because it increases for the angles close to the boundaries of the measurement range.

Figure 15. Mean absolute value of the flow angle error as a function of the Reynolds number for the cylindrical, the trapezoidal and the cobra type probes, $\delta=60^{\circ}$.

\section{Pitch Angle Effects}

Deviations from the zero pitch angle $\beta$ (angle between the flow and the probe measurement plane) are a classical source of inaccuracy in the regular operation of THP probes. Typically, it is considered that the measurements are accurate enough if pitch angle misalignments do not exceed $10^{\circ}$ or $12^{\circ}([4],[10])$. Concerning the extended angular range, it is expected that deviations of the flow from the measurement plane will have a major effect on the probe performance that in the traditional range. Also, this effect depends on the probe head geometry. To analyze the sensitivity of the cylindrical, the trapezoidal and the cobra type probes to pitch deviations, an additional set of measurements was conducted in the calibration facility varying the pitch angle from $0^{\circ}$ to $20^{\circ}$ at intervals of $2.5^{\circ}$. The construction angle of the probes is $60^{\circ}$, for a constant Reynolds number of $1.8 \times 10^{4}$.

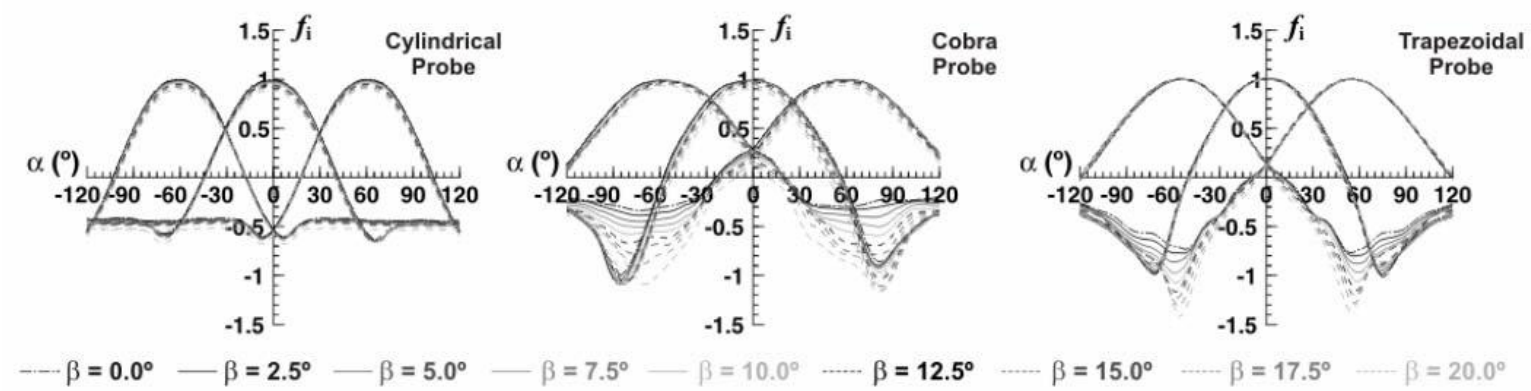

Figure 16. Pressure coefficients of the cylindrical, the trapezoidal and the cobra type probes as a function of the pitch angle, $\delta=60^{\circ}$.

The distributions of the pressure coefficients obtained for the three probes are shown in figure 16. The observed general trend is that the pressure coefficient is continuously reduced as the pitch angle increases. For the cylindrical probe the variations are significant only if the pitch 
angle is higher than $15^{\circ}$, with similar values all along the yaw angular range. Variations with the pitch are more pronounced in the case of trapezoidal and cobra type probes. Though important differences are only noticeable when the pitch angle exceeds $12.5^{\circ}$ in the traditional yaw range, in the extended intervals the impact is already appreciable for pitch deviations of only $2.5^{\circ}$ (especially in the fully-detached zones). This impact is particularly intense in the lateral holes, rather than in the central one. Besides, for $\beta=17.5^{\circ}$ and $20^{\circ}$ they are more evident for trapezoidal than for cobra type probes.

Figure 17. Mean absolute value of the flow angle error as a function of the pitch angle for the cylindrical, the trapezoidal and the cobra type probes, $\delta=60^{\circ}$.

To compare the sensitivity of the pressure probes to pitch deviations, the error in the determination of the yaw angle is plotted in figure 17. To that end, the calibration corresponding to the $\beta=0^{\circ}$ condition has been employed as the baseline reference to reduce data measured at other pitch values. The figure represents the mean absolute value of the flow angle error, in a similar fashion to the one adopted to obtain previous figure 15. Once again, the interval considered for the yaw angle is the operative range of the cylindrical probe $\left( \pm 60^{\circ}\right)$. If a higher angular range would have been considered, a larger error would have been also obtained for cobra type and trapezoidal geometries, because it increases as yaw angle increase. The line corresponding to the cylindrical probe has a smooth slope, with mean error not exceeding $0.3^{\circ}$ for pitch deviations lower than $10^{\circ}$. Higher misalignments lead to superior mean errors, reaching up to $0.8^{\circ}$ when $\beta=20^{\circ}$. Results corresponding to trapezoidal and cobra type geometries present stepper slopes. Up to $15^{\circ}$ the error is quite similar, but a bit lower for the cobra type design than for the trapezoidal one. The cobra type probe reaches the maximum mean error $\left(1.6^{\circ}\right)$ for a $15^{\circ}$ pitch angle, which is further maintained quite constant for higher pitches. Conversely, the mean error for the trapezoidal probe is progressively increased, being as high as $2.5^{\circ}$ for $\beta=20^{\circ}$.

\section{Conclusions}

The effect of the different head geometries of pneumatic THP probes on the measurement angular range and the flow variables uncertainty has been analyzed in this paper. Besides, the sensitivity of their performance to variations in the Reynolds number and pitch misalignments has been addressed. For that purpose, three different geometries have been considered in the study: cylindrical, trapezoidal and cobra type probes, with a separation angle of $60^{\circ}$ between the holes. In the case of cylindrical and cobra type probes, the effect of the construction angle on both the measurement angular range and the uncertainty transmission has been also explored, using additional probes with construction angles of $30^{\circ}$ and $45^{\circ}$. 
The following conclusions can be drawn from the present investigation:

- Cobra type probe presents the largest measurement range, followed by the trapezoidal and the cylindrical geometries. The limit of the angular range is due to the emergence of double points in all of them. This means that the extended angular range cannot be further enlarged, even if any other data reduction procedure is employed.

- For the cylindrical probe the angular range is higher if the separation angle between the holes is $30^{\circ}$. If this is the case, the restriction of the angular interval is imposed by duplicated zones, instead of the double points limitation when $\delta=45^{\circ}$ and $60^{\circ}$. Therefore, it is not possible to enlarge the angular range in any case. For the cobra type probe the higher angular range is attained when the construction angle is $45^{\circ}$. This geometry is restricted by double points when the separation angle between the holes is $60^{\circ}$. However, when $\delta=30^{\circ}$ or $45^{\circ}$ the limitation is because the arising of singular points, so it would be possible to enlarge the operative range introducing other data reduction procedure, for example, a three-zone method.

- Within the extended angular range the transmission of uncertainty to the flow variables is lower with the cylindrical probe than with the other two geometries. It has been observed that both cylindrical and cobra type probes increase the uncertainty when the angular separation between their holes is reduced.

- As expected, the cylindrical design is more stable to Reynolds number variations, while the trapezoidal probe presents the highest sensitivity to changes in the velocity magnitude. In all the geometries tested, the errors introduced in the determination of the yaw angle increase in the extended region of the angular range. A trapezoidal probe with a construction angle of $30^{\circ}$ or $35^{\circ}$ should present a better performance in these conditions, because the fullydetached regions in the lateral holes are displaced towards the boundaries of the angular interval.

- The cylindrical probe is also more stable to flow misalignments with respect to measurement plane, while the trapezoidal design is newly more sensitive to pitch angle deviations. For all the geometries the error derived from this effect increases as both yaw and pitch angles increase.

- Finally, as a general rule, it is assumable that all these types of pneumatic probes are less sensitive to variations in the Reynolds number than to pitch angle misalignments.

\section{Acknowledgements}

This work was supported by the Research Project "Effect of the volute geometry of centrifugal pumps on the fluid-dynamic perturbations due to rotor-stator interaction", ref. DPI-2006-15638C02-01, MEC.

\section{Nomenclature}

$\begin{array}{ll}\text { THP } & \text { Three-Hole Probe } \\ C_{\alpha} & \text { Angular coefficient } \\ C_{\mathrm{p}} & \text { Pressure coefficient } \\ C_{\mathrm{Po}} & \text { Total pressure coefficient } \\ C_{\mathrm{Ps}} & \text { Static pressure coefficient } \\ f_{\mathrm{i}} & \text { Pressure coefficient } \\ I_{\alpha} & \left.\text { Angle uncertainty, }{ }^{\circ}\right] \\ I_{\mathrm{P}} & \text { Pressure uncertainty, }[\mathrm{Pa}] \\ I_{\mathrm{Pd}} & \text { Dynamic pressure uncertainty, }[\mathrm{Pa}] \\ I_{\mathrm{Ps}} & \text { Static pressure uncertainty, }[\mathrm{Pa}] \\ P_{\mathrm{i}} & \text { Pressure measurement, }[\mathrm{Pa}] \\ P_{\mathrm{d}} & \text { Dynamic pressure, }[\mathrm{Pa}] \\ P_{0} & \text { Total pressure, }[\mathrm{Pa}]\end{array}$




$$
\begin{array}{ll}
P_{\mathrm{s}} & \text { Static pressure, }[\mathrm{Pa}] \\
\mathrm{Re} & \text { Reynolds number }
\end{array}
$$

Greek letters

$\alpha \quad$ Flow angle (yaw angle), $\left[^{\circ}\right]$

$\beta \quad$ Flow angle (pitch angle), $\left[{ }^{\circ}\right]$

$\delta \quad$ Construction angle of the probe, $\left[{ }^{\circ}\right]$

$$
\begin{aligned}
& \text { Subscripts } \\
& \mathrm{i}
\end{aligned}
$$

Superscripts

Derivative

\section{References}

[1] Argüelles Díaz K M, Fernández Oro J M and Blanco Marigorta E 2008 Direct calibration framework of triple-hole pressure probes for incompressible flow Measurement Science and Technology 19075401 (13pp)

[2] Dudziniski T J and Krause L N 1971 Effect of inlet geometry on flow-angle characteristics of miniature total-pressure tubes NASA TN D-6406

[3] Erwin J R 1964 Experimental Techniques. Section D of Aerodynamics of Turbines and Compressors (Princeton University Press)

[4] Bryer D W and Pankhurst R C 1974 Pressure-probe methods for determining wind speed and flow direction (National Physical Laboratory, ISBN: 011-480012X)

[5] Chue S H 1975 Pressure probes for fluid measurement Progress in Aerospace Sciences 16(2) $147-223$

[6] Gameiro M C, Pereira C A C and Cruz J M S 2001 On the use of a linear interpolation method in the measurement procedure of a seven-hole pressure probe Experimental Thermal and Fluid Science 28 1-8

[7] Milanovic I M and Kalkhoran I M 2000 Numerical calibration of a conical five-hole probe for supersonic measurements Meas. Sci. Technol. 11 1812-1818

[8] Zilliac G G 1989 Calibration of seven-hole pressure probes for use in fluid flows with large angularity NASA TM 102200

[9] Everett K N, Gerner A A and Durston D A 1983 Seven-hole probe for high angle flow measurement: Theory and Calibration AIAA J. 21 992-8

[10] Dudziniski T J and Krause L N 1969 Flow-direction measurement with fixed-position probes NASA TM $X-1904$

[11] Lewis W E 1966 Fixed-direction probes for aerodynamic measurements. Proc. Inst. Mech. Eng. 180 141-52

[12] Treaster A L and Yocum A M 1979 The calibration and application of five-hole probes ISA Transactions 18 23-34

[13] Sumner D 2002 A comparison of data-reduction methods for a seven-hole probe Journal of Fluids Engineering 124 523-7

[14] Ligrani P M, Singer B A and Braum L R 1989 Miniature five-hole pressure probe for measurement of three mean velocity components in low-speed flows J. Phys. E: Sci. Instrum 22 868-76

[15] Hooper J D and Musgrove A R 1997 Reynolds stress, mean velocity, and dynamic static pressure measurement by a four-hole pressure probe Experimental Thermal and Fluid Science 15 375-83

[16] Guo Y and Wood D H 2001 Instantaneous velocity and pressure measurements in turbulent mixing layers Experimental Thermal and Fluid Science 24 139-50

[17] Chen J, Haynes B S and Fletcher D F 2000 Cobra probe measurements of mean velocities, Reynolds stresses and higher-order velocity correlations in pipe flow Experimental Thermal and Fluid Science 21 206-17 
[18] Kupferschmied P, Köppel P, Gizzi W, Roduner C and Gyarmathy G 2000 Time-resolved for measurements with fast-response aerodynamic probes in turbomachines Meas. Sci. Technol. 11 1036-54

[19] Humm H J, Gizzi W P and Gyarmathy G 1994 Dynamic response of aerodynamic probes in fluctuating 3D flows Proc. 12th Symposium on Measuring Techniques for Transonic and Supersonic Flows in Cascades and Turbomachines (Prague)

[20] Sieverding C H, Arts T, Dénos R and Brouckaert J F 2000 Measurement techniques for unsteady flows in turbomachines Experiments in fluids 28 258-321

[21] Weidman P D 1968 Wake transition and blockage effects on cylinder based pressures (Ph.D. Thesis: California Institute of Technology)

[22] Argüelles Díaz K M, Fernández Oro J M and Blanco Marigorta E 2008 Three-hole pressure probes at large Proceedings of XIXth Symposium on Measuring Techniques for Transonic and Supersonic Flows in Cascades and Turbomachines (Belgium)

[23] Smout P D and Ivey P C 1997 Investigation of wedge probe wall proximity effects: part 1 - experimental study ASME Journal of Engineering for Gas Turbines and Power 119 598-604

[24] Smout P D and Ivey P C 1997 Investigation of wedge probe wall proximity effects: part 2 - numerical and analytical modeling ASME Journal of Engineering for Gas Turbines and Power 119 605-611

[25] Argüelles Díaz K M, Fernández Oro J M and Balnco Marigorta E 2008 Cylindrical threehole pressure probe calibration for large angular range Accepted for publication in Flow Measurement and Instrumentation

[26] Argüelles Díaz K M, Fernández Oro J M and Blanco Marigorta E 2008 Extended angular range of a three-hole cobra pressure probe for incompressible flow Journal of Fluids Engineering 130101401 1-6

[27] Dominy R G and Hodson H P 1993 An investigation of factors influencing the calibration of five-hole probes for three-dimensional flow measurements ASME Journal of Turbomachinery 115 513-519 
This document is a pre-print version of the scientific paper published by Elsevier. It has been released by the authors to fulfill all the publisher requirements established for Article Sharing: https://www.elsevier.com/about/policies/sharing

\section{(9) $\Theta \Theta \Theta$}

(C) 2019. This manuscript version is made available under the Creative Commons Attribution-NonCommercial-NoDerivatives 4.0 International License (CC-BY-NC-ND 4.0 license) http://creativecommons.org/licenses/by-nc-nd/4.0/ 